\title{
The Domino Effect
}

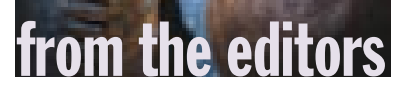

\section{Charlene O'Hanlon, ACM Queue}

S ometimes, the undertaking of a task leads to a result far different from the expected. Any homeowner can attest to that.

Case in point: About four years ago, after living in my cozy little cape for about two years, I decided to replace the toilet in the upstairs bathroom because it had not been installed properly, which made me nervous each time it was used. How it survived 20 years I will never know, but I was adamant that it needed to be replaced.

Keeping in mind that the upstairs bathroom had not been renovated in about 20 years, you can imagine the color scheme. The sink and toilet were both a medium gray-a color I know for a fact is no longer available. So when the search was under way for a replacement model that would match the existing décor, I discovered very quickly that I would have a better chance of being elected mayor of my little village. I settled on white and thought that was the end of it.

But no. With a gray sink and white toilet, the bathroom looked strangely mismatched. The sink, which was a small thing that hung from the wall and had no base, was ready for retirement as well, so the decision was made to replace it with a nice, compact, pedestal model.

Replacing the sink was quickly followed by replacing the shower stall (a decision I'm not sure was the correct one in hindsight), the flooring, and removing the tiles on the wall, which turned out to be those vinyl four-foot-bysix-foot sheets that are glued to the wall and are supposed to look like tile. The wall had been painted so I had no idea my wall tiles were imposters. Removing those sheets was extremely difficult, and once the sheets had been pulled off and the glue residue removed, the wall was literally beyond repair. So off came the old drywall (and the ceiling as well, since it also was in rough shape).

The project by then had reached the point of no return. Fast forward, and today I have a new toilet, new sink, new medicine cabinet, new shower, new ceramic tile floor, new moldings, a stunning coat of paint on the new walls, new light fixtures, and lovely new hardware. The entire process took three years, but it finally became the bathroom I wanted it to be. Starting from a simple toilet replacement, the domino effect had forced a complete

\section{Sometimes, ADDING}

ONE COMPONENT

CAN LEAD TO A TOTAL

NETWORK REDESIGN overhaul of my bathroom (did I mention the pipes had to be reset as well, which meant part of the floor had to be removed?).

It's not uncommon to start with one goal in mind and end up with something much different due to forces beyond our control or grandiose ideas-it's part of the learning process. In technology, however, the domino effect can lead to major changes in a network, sometimes to the detriment of the network. Luckily, that event is becoming less of a common occurrence thanks to the onset of Web services. More than a temporary solution to a permanent problem, Web services have emerged as the cleanest way to get multiple applications to talk to each other and swap data. Hallelujah, I say, for the open standards that make Web services so effective.

Just take a look at those companies that have successfully implemented Web services: Google, Amazon, PayPal, FedEx...the list goes on. In fact, Amazon CTO Werner Vogels, who was one of our January Queuecast guests (go online to hear the interview), dissects his company's approach to Web services in this month's interview.

Also in this issue, Krishna Sankar of Cisco Systems offers additional integration food for thought in his article, "The Network's New Role." And for takes on a few interesting side issues, check out the articles penned by Amazon's Felipe Cabrera and Jeff Barr ("AI Gets a Brain") and Ryan Barrows of Morgan Stanley ("Search Considered Integral"). Both of these articles are chock full of ideas worth considering.

Web services can save IT administrators a lot of agita, allowing services and components to be reused within an infrastructure. Without Web services, using noninteroperable applications could eventually lead to a total-and possibly unnecessary—system redesign. Just like one toilet can lead to a complete bathroom renovation. $Q$

CHARLENE O'HANLON, editor of ACM Queue, is ready to tackle just about any home improvement job, as long as it doesn't require a blowtorch (there's a thin line between confidence and hubris). 